\title{
Semantic Segmentation of Cell Nuclei in Breast Cancer using Convolutional Neural Network
}

\author{
Tomiya Said Ahmed Zarbega \\ Department of Computer Engineering \\ Kastamonu University, Kastamonu, Turkey
}

\author{
Yasemin Gültepe \\ Department of Computer Engineering \\ Kastamonu University, Kastamonu, Turkey
}

\begin{abstract}
Many studies have been carried out in the literature and practice by using deep learning technique and successful results have been obtained. Convolutional Neural Network $(\mathrm{CNN})$, a specialized architecture of deep learning, is particularly successful in image processing. Semantic segmentation is a computer vision task to estimate pixel tags corresponding to the region to which it belongs or to the region of the surrounding region. Semantic segmentation aims to understand the class of special objects in the scene. In this paper, Convolutional Neural Network based on detection and semantic segmentation of cell nuclei for breast cancer was performed on the "PSB 2015 crowdsourced nuclei" data set. As a result, the CNN model gave the highest performance with precision (0.844), recall (0.832) and accuracy (0.851) compared to other classifiers in the literature and the most advanced methods.
\end{abstract}

\section{Keywords}

Convolution neural network, image segmentation, semantic segmentation, nuclei cells, breast cancer.

\section{INTRODUCTION}

Breast cancer poses a serious danger to women's health. Breast cancer is the result of the cells gaining cancer qualities (unlimited and uncontrolled growth and growth). Breast cancer, which is extremely widespread cancer in women in the world and in our country, occurs in one to every 8-10 women throughout life.

Although the rates of death of patients as a result of breast cancer have decreased in recent years, they are still high. Early diagnosis and treatment is possible by conducting screening programs in breast cancer. Early diagnosis in breast cancer is of great importance in increasing survival and success of treatment. Early identification methods in breast cancer: Breast examination by yourself, clinical breast examination and mammography. Mammography is an important screening method that reduces mortality in breast cancer by $30 \%$. Mammography provides early detection of tumors that have no clinical findings yet [1].

Digital image, unlike analog photo, is the image consisting of sequences composed of pixels, each of which has independent value, in the physical environment. There was a lot of useful information in the image. Computer-aided detection and diagnosis systems pass medical images through a number of stages. These stages are listed as image pre-processing, segmentation, feature extraction and classification. The performance of the segmentation process in the image recognition process significantly affects the image recognition success. Image segmentation can be explained as distributing an image into significant regions in which dissimilar characteristics are kept. For instance, there may be comparable brightness in the image, and this brightness can describe objects in dissimilar fragments of the image.

Semantic segmentation is the effort of clustering together fragments of an image belonging to the uniform class of objects. It is a form of pixel-level estimation, because each pixel in the image is classified by a category.

The image semantic segmentation challenge consists of the classification of each pixel of an image (or just a few of them) corresponding to an object, each object (or category) corresponding to an object or part of the image. Semantic image segmentation has multiple operations. For example, identifying road signs, colon crypts segmentation, ground use and ground cover classification. With the popularity of deep learning in recent years, many semantic segmentation problems, a great difference in accuracy and efficiency, and deep architectures that surpass other approaches are mostly addressed using Convolutional Neural Networks [2].

Deep learning is a special subfield of machine learning, which is the concept of artificial neural networks and deals with algorithms stimulated by the usability of human brain cells called neurons. The main advantage of Deep Learning is that by pasting basic feature sets such as edges or corners, it creates complex concepts from simple raw material data [3].

Deep learning algorithms have solved a variety of computer vision tasks with an increasing level of difficulty. The most popular deep learning algorithms can be listed as follows: Convolutional Neural Network $(\mathrm{CNN})$, Recurrent Neural Networks (RNNs), Long Short-Term Memory Networks (LSTMs), Stacked Auto-Encoders, Deep Boltzmann Machine (DBM), Deep Belief Networks (DBN), etc. [4].

In this paper, the semantic segmentation method with $\mathrm{CNN}$ is used for recognition and segmentation of the nuclei cells in breast cancer. One of the main functions in the proposed work is detect the core of breast cancer nuclei, then extract the regions of interest (ROI). ROI usually mean meaningful and important regions in images. The use of ROI can prevent the processing of flipping image points and speed up processing [5]. To achieve the purpose of this step, CNN is applied to histological images. At the end of each step the ground truth being compared with the extracted ROI.

In this paper, information about the literature review is given in the second section after the introduction. In the third section, the data set used and the proposed methodology are explained. Then, in the fourth section, performance measurement calculation was performed for segmentation of the nuclei image. In the last section, results and evaluations about the study are included. 


\section{LITERATURE REVIEW}

There are approximately 1.38 million new cases of breast cancer each year. While the use of deep learning techniques for the diagnosis of cancer in the clinical field is increasing, there are several studies that facilitate the detection of breast cancer. In this section, some important studies focusing on the detection and classification of breast cancer nuclei are described using $\mathrm{CNN}$ in the literature.

It is vital that the histopathological images of breast cancer are automatically classified as benign and malignant cancer with the aid of computer-aided diagnostic systems, and early treatment is therefore essential. Rakhlin et al. reported an $87.2 \%$ classification success for the classification task of 4 classes using strong data enhancement and deep convolutional features extracted from different scales with CNNs to classify Hematoxylin and Eosin (H\&E) stained histological breast cancer images from a dataset consisting of $400 \mathrm{H} \& \mathrm{E}$ stain images [6]. For the classification task of 2 classes to detect carcinomas, they achieved $93.8 \%$ accuracy at the high precision work point, average (AUC) $97.3 \%$ area under the ROC curve and $96.5 \%$ sensitivity and $88.0 \%$ specificity value. These values reveal the performance of the model made with CNN.

Lévy and Jain created three different CNN architectures for the breast mass classification on the Digital Database for Screening Mammography [7]. As a result of the GoogLeNet (Aug-Large Context) model, a high performance was achieved with Accuracy (0.929), Precision (0.924) and Recall (0.934).

Cruz-Roa et al. used the CNN model for automatic detection and visual analysis of invasive ductal carcinoma (IDC) tissue areas on all slide images of breast cancer. 3 fully connected layers were used, 16 neurons were used for the first convolution pooling layer, 32 for the second convolutional pooling layer and 128 neurons for the fully connected layer. For all experiments, a fixed convolution core of $8 \times 8$ and maximum pooling of $2 \times 2$ were applied. Precision $65.40 \%$, Sensitivity $79.60 \%$, Specificity $88.86 \%$, F1 measurement $71.8 \%$ and balanced accuracy $84.23 \%$ achieved success in determining IDC tissue regions on the data set consisting of 169 images obtained from 162 female patients diagnosed with IDC [8].

Sirinkunwattana et al. Used Spatial Constrained Convolutional Neural Network (SC-CNN) for core detection. A new Neighbor Community Predictor (NEP) combined with CNN was used to accurately estimate the class labels without the need to divide the detected cell nuclei. As an introduction to the SC-CNN deep learning model, a large colorectal adenocarcinoma images of more than 20,000 annotated cores reported classification accuracy of up to $80.20 \%$ on images in the dataset [9].

Wang et al. used CNN model on AMIDA13 dataset and ICPR12 datase for the detection of mitosis. They created a deep neural network consisting of 3 layers containing 64, 128 and 256 neurons, respectively. For each layer, a fixed $8 \times 8$ convolution core and a $2 \times 2$ combining core were used. An IC measurement rate of 0.7345 was obtained on the ICPR 12 mitosis data set. In addition, experiments in the AMIDA13 dataset show that it is still necessary to increase the accuracy of the presented approach. [10].

Cruz et al. obtained $89.4 \%$ in F-measure and $91.4 \%$ in balanced accuracy by using deep learning architecture for basal cell carcinoma cancer detection in a data set consisting of 1,417 images from 308 interests of skin histopathology slides [11].

Wan et al. presented a new approach to image analysis to automatically classify low, medium and high degrees of breast cancer in digitized histopathology. [12]. Using segmentation method based on hybrid active contour model; To create a set of semantic level properties, merged image attributes, a CNN model was created that obtained the proportions of nuclei of different degrees, along with pixel level (texture) and object level (architecture) properties.

It is very difficult to detect the cell nuclei and classify them in the separation of the cell nuclei within the tissue structure where the H\&E stained Histological images are focused. Cireșan et al. achieved a success rate of Precision $88 \%$, Recall $70 \%$, and F1 Score $78.2 \%$ at the pixel level in the public MITOS dataset (Mitosis Detection in Breast Cancer Histological Images) containing 50 images corresponding to 50 high-power domains in 5 different biopsy slides stained with Hematosin and Eosin [13].

\section{DATASET AND PROPOSED METHODOLOGY}

\subsection{Dataset}

As general tissue paint; It is the Hematoxylin-Eosin dye that is used in the separation of nucleus and cytoplasm, which has the widest use among histological dyes, and paints different parts of the tissue differently. Hematoxylin is generally; It shows the intranuclear detail well by painting the nucleus in blue-black color. Eosin Hematoxylin-Eosin; paints cell cytoplasm and connective tissue elements in various variations in pink, orange and red [14]. In Figure 1, an example of tissue painted with $\mathrm{H} \& \mathrm{E}$ is given.

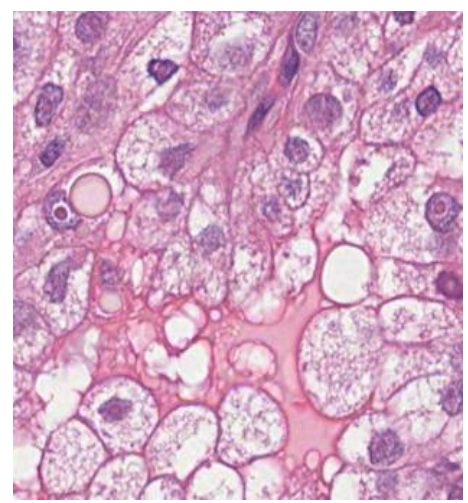

Fig 1: A sample nuclei image from breast cancer

As seen in Figure 1 the boundary of the nuclei is not so clear. For this reason, the extraction of the nuclei is so difficult task in the segmentation step. For example, the sample region in Figure 1 is shown in Figure 2.

As shown in this Figure 2 the boundary between two nuclei is so difficult to detection and this is big problem in nuclei cells detection and segmentation.

In this study, CNN based experiences are proposed using the popular, free and public PSB 2015 crowdsourced core data set [15]. From this data set, 810 microscopic hematoxylin and eosin stained breast biopsy samples were used. Each tile comes from a different patient. 


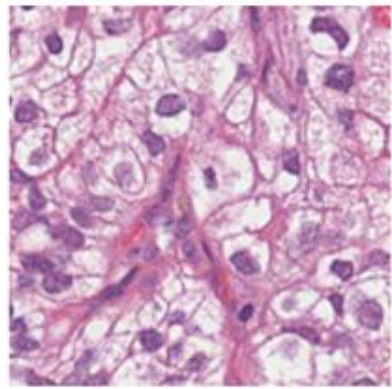

(a)

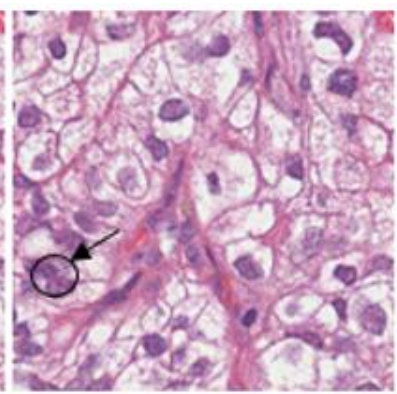

(b)
Fig 2: (a) Original nuclei image and (b) difficult boundary place

The data set contains nuclei detection, nuclei segmentation and original images. These data set contains 810 images from 810 peoples. It involves a total of 810 labeled cell nuclei in all dataset images and the size of each image in each set is $400 * 400$. Each kernel of images in the data set is one of four kernel types. These types are labeled as colon, breast cancer, kidney and others. Examples of the types are shown in Figure 3. The "Others" label indicates nuclei belonging to the nucleus type other than colon, breast and kidney cancer.

Colored image were not used in this study and the gray level image were used. Because the processing in color image need long time. Also working on one channel is better than working on three channel.

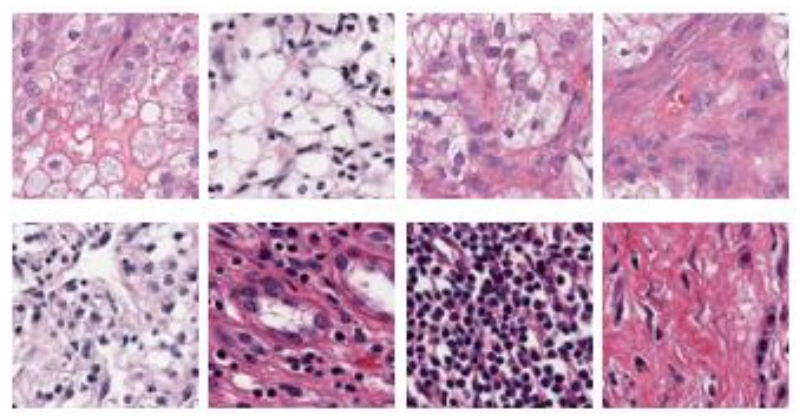

Fig 3: Sample images of the dataset

\subsection{Semantic Segmentation}

The purpose of semantic image segmentation is to annotation each image. Semantic segmentation is a natural step in moving from rough to subtle implications. The source can be found in the classification, which consists of making an estimate for the entire input. The next step is localization or detection, which provides not only classes but also additional information about their spatial location. Finally, semantic partitioning makes fine-grained inferences by making intense predictions that generate tags for each pixel, so each pixel is labeled with the class of the object ore region around it [16].

In this study, semantic segmentation was used to label core cells and non-core cells from the core image dataset. Labeling process has been carried out with deep convolutional neural networks, which are out of the classical methods used in the labeling of the data set, and which have impressive results in the semantic segmentation of the images in recent years.

\subsection{Performance Criteria}

There are several measurement types based on standard measurement uses to compare the segmentation image obtained based on the use of the confusion matrix, such as Accuracy, Precision, Accuracy and Specificity [17]. Dice
Criterion is categorized under similarity measurements.

The confusion matrix is a table designed to label the performance of the classification model by showing the criteria of the "strict reference" and the output of the segmentation model. The confusion matrix is as follows for binary classification [18]:

\begin{tabular}{|c|c|c|c|}
\hline & \multicolumn{2}{|c|}{ Segmentation Output } \\
\hline & & Positive & Negative \\
\hline 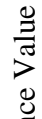 & $\begin{array}{l}\stackrel{D}{*} \\
0 \\
0 \\
0\end{array}$ & TP & $\mathrm{FN}$ \\
\hline 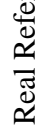 & 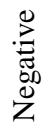 & FP & $\mathrm{TN}$ \\
\hline
\end{tabular}

Fig 4: Confusion matrix

What can be learned from the confusion matrix in Figure 4 ? The answer to this question can be given as follows [16]:

True Positive (TP): Pixels that belong to the positive class and are correctly included in the positive class.

True Negative (TN): Pixels that belong to the negative class and are correctly included in the negative class.

False Positive (FP): Pixels that belong to the negative class and are incorrectly included in the positive class.

False Negative (FN): Pixels that belong to the positive class and are incorrectly included in the negative class.

There are some proportions calculated from the confusion matrix. These terminologies showing the relationship of TP, TN, FP and FN are given below.

Total $=T P+T N+F P+F N$

Real Positives $=T P+F N$

Real Negatives $=T N+F P$

All this situation is shown in Figure 5. The segmented region is shown by dark blue colored and reference region is shown by red colored lines [19].

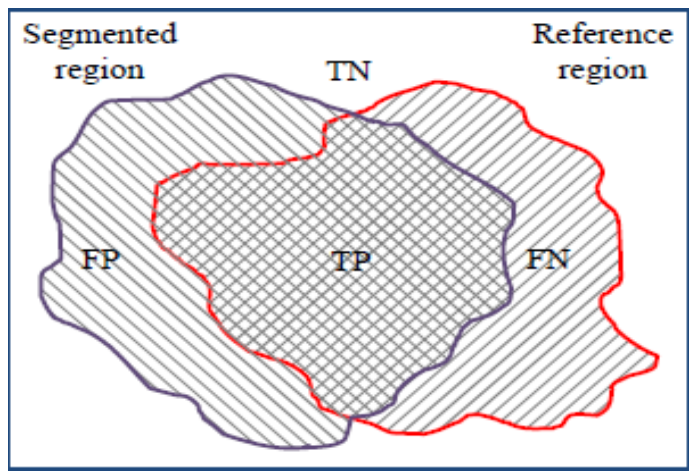

Fig 5: Segmented region and reference region

In TPR and FPR notation; TP shows positive pixels properly labeled, FP shows false negative pixels labeled positive, FN shows positive pixels labeled incorrectly, and $\mathrm{TN}$ is correctly labeled negatively. 
The false negative ratio (FNR) is shown in following equation:

$F N R=\frac{F N}{P}=\frac{F N}{F N+T P}=1-T P R$

The false positive ratio (FPR) is shown in following equation:

$F P R=\frac{F P}{N}=\frac{F P}{F P+T N}=1-T N R$

Below is a brief description of the above-mentioned measure types, which are more common in segmentation:

Sensitivity: The test is the ability to distinguish patients from real patients.

Specificity: The test is the ability to separate the solid from the real solid.

Accuracy: When it is desired to obtain a single measurement by combining sensitivity and specificity, one of the measurements used is the possibility of correct test results. In fact, the test is called patient and robust total accurate diagnostic rate.

The sensitivity (TPR), specificity (TNR) and accuracy (ACC) are shown as equation (6), (7) and (8) respectively [20].

$$
\begin{aligned}
& \text { Sensitivity }(T P R)=\frac{T P}{T P+F N} \\
& \text { Specificity }(T N R)=\frac{T N}{T N+F P} \\
& \text { Accuracy }(A C C)=\frac{T P+T N}{T P+F N+T N+F P}
\end{aligned}
$$

DICE similarity coefficient is a spatial overlap based criterion. Spatial overlap-based metrics do not measure the segments of the segmented image in line with the basic true image, but measure the extent to which pixels of the segmented image are correctly detected as foreground or background.

Considering $\mathrm{A}$ as the nonzero pixels in the true image and nonzero pixels in the B segmented image [21, 22]:

$D I C E=\frac{2 *|A \cap B|}{|A|+|B|}=\frac{2 T P}{2 T P+F P+F N}$

The results obtained are between $0-1$, approaching the result shows that the segmentation performance is very good.

\subsection{Convolutional Neural Network}

Evolutionary neural networks are a sub-branch of deep learning and are often used to analyze visual information. Common areas of use are image and video recognition, suggestion systems, image classification, medical image analysis, and natural language processing. CNNs are a special Multilayer Neural Networks (MNN) type. The major advantage of $\mathrm{CNN}$ is its shared weight usage and local connectivity. Input data can be used to recognize local features used to recognize dermoscopy images and to label pixel by pixel. CNN can abstract the information of objects and reduces its size. In addition, the $\mathrm{CNN}$ abstraction and classification process uses the same deep architecture for optimization of the same parameter in different steps, it can effectively improve learning ability [23, 24].

CNN includes many layers that can be fed forward, and which are different from neural networks, as well as a featureenhancing layer. CNNs consist of two basic components. These components are attribute extraction and classification. The feature extraction component performs a group of convolution and pooling processes to determine the features. The classification component, on the other hand, produces probability estimates in the image taken as input based on the attributes obtained. The images given to $\mathrm{CNN}$ progressed between the layers of the model within the framework of certain rules and feature inferences were realized and recognition process was realized as a result of the operations in the classification layer [25].

ESA consists of the following layers: Input Layer, Convolution Layer, Flattened Linear Unit Layer (RELU), Pooling Layer, Fully Connected Layer, and Dropout Layer and Classification Layer consists of. The number of layers used can be changed by the person who designed the model.

The structure of a convolutional neural network is shown in Figure 6. The network has two convolution layers, two subsampling layers following the convolution layers, and a fullylinked layer on the top layer.

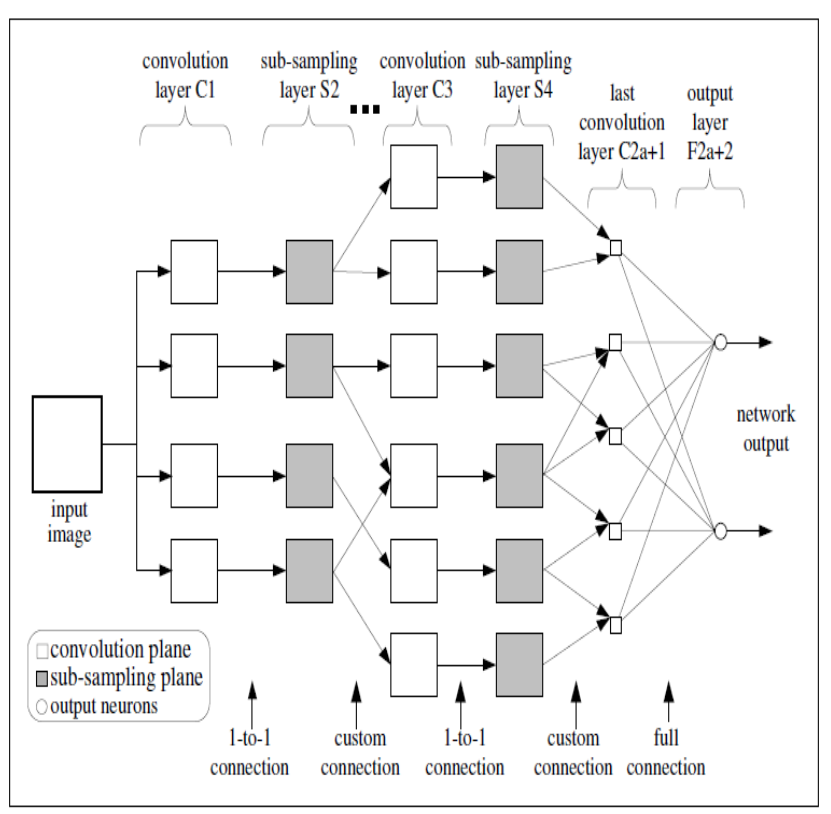

Fig 6: Typical convolutional neural network structure

In this structure, the information of the image is apply to the convolutional layer, then filter is applied to each part. After the filter process, the features of the image obtained from the last convolutional layer. The pixels obtained as a result of this process are interpreted and the problem is tried to be solved [26].

The CNN architecture presented in this study has three main layers where each main layer contains three sublayers, which will be the input layer and a total of ten layers. First main layer contains convolution layer and rectified linear unit and maxpool. Second main layer contains convolutional layer rectified unit and transposed convolution layer. The transferred convolution is also known as the non-convoluted layer. The deconvolution layer reverses the operation of the standard convolution layer, restoring the original input. Last main layer contains convolutional layer, softmax and classification layer. Applying softmax to a vector is done by overlapping each element and then normalizing the vector using the L1 norm (sum of absolute values) so that values can be added up to 1 and interpreted as probability.

\subsection{Mathematical Morphology}

Mathematical morphology has become one of the frequently used and researched methods in image analysis. Mathematical morphology uses mathematical principles to distinguish between object and background in an image. Mathematical morphology is based on the structural properties of the object 
in the image [27].

RGB images contain much more semantic information about objects than gray-level images. It is decomposed into RGB image bands to perform morphological processing on RGB images.

\subsection{Methodology}

The flowchart of the proposed method is shown in Figure 7. The model proposed in our study consists of five basic steps: 1.Initialization, 2.Implement the $\mathrm{CNN}$ on the Image, 3.Morphological operations, 4.Binarization and segmentation and 5.Nuclei segmentation and accuracy validation.

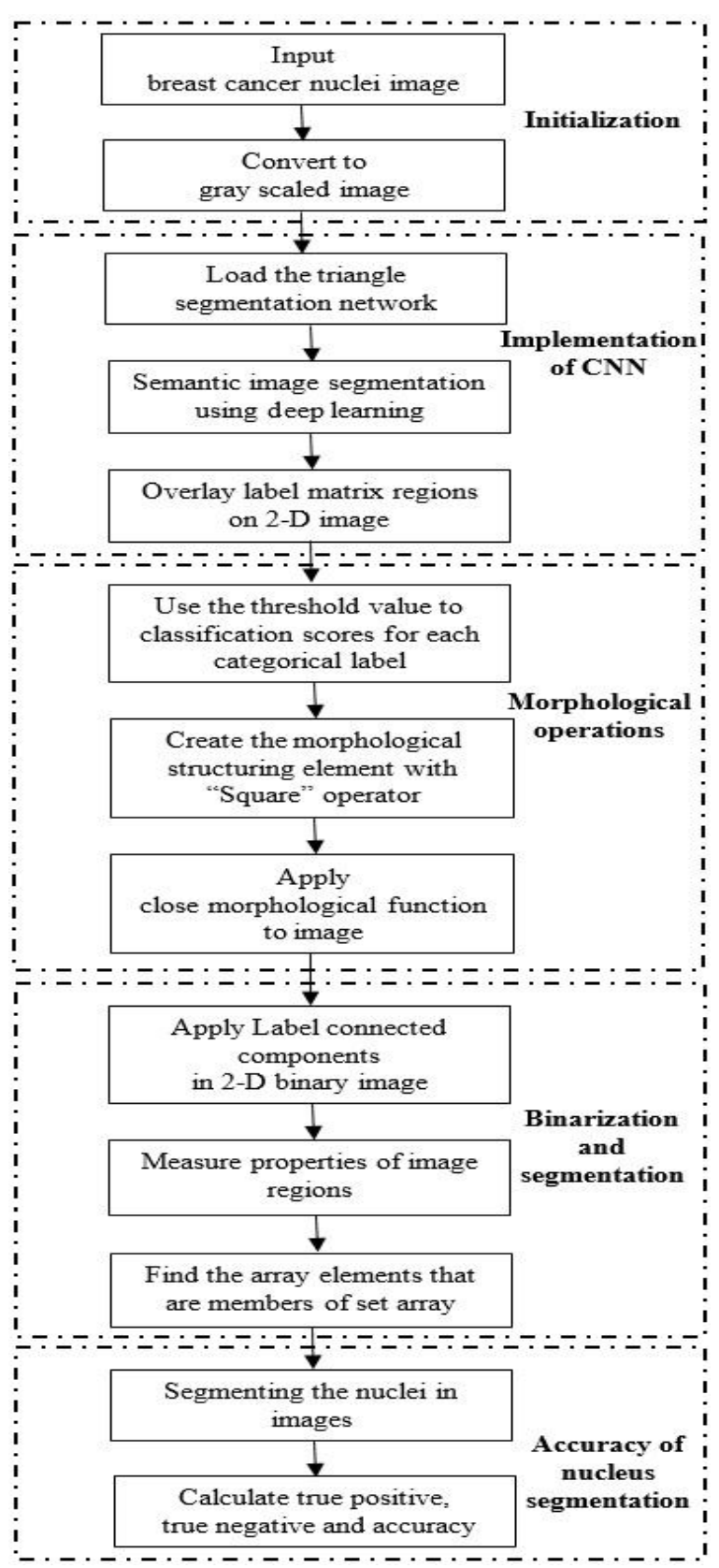

Fig 7: Flowchart of the proposed method

Initialization: In this step the breast cancer nuclei image is import to the matrix. Then the image convert to Gray scaled image.

Implement the CNN on the Image: Here we load the triangle segmentation network for using in the semantic image segmentation based on deep learning method. Then we overlay label matrix regions on 2-D image.

Morphological operations: In this step we use the threshold value to classification scores for each categorical label. Thresholding methods are among the most used techniques for extracting information from the image using digital image processing techniques. Thresholding is one of the simplest approaches to image segmentation. The basic logic of the thresholding method is to separate the object in the image and the background of the image based on different intensity values [28]. Then morphological structuring element was created with the "Square" operator. Finally, a morphological function close to the image is applied.

Binarization and Segmentation: In this step we apply label connected components in 2-D binary image. Then the properties of the image regions are measured and the array elements that are members of the set array are found.

Nuclei segmentation and Accuracy validation: This step is last step and here the nuclei image is segmented. The threshold value is used to classification scores for each categorical label. In this method there is no any thresholding value that use for nuclei image segmentation. We used semantic segmentation for extraction of the nuclei from image. For post processing the morphological structuring element with "Square" operator is created and then apply close morphological function to image. Finally, true positive, true negative and accuracy are calculated.

\section{PERFORMANCE MEASURE CALCULATION FOR SEGMENTATION OF NUCLEI IMAGE}

In order to show the performance of proposed method, three parameters are applied to analysis of the method. These parameters are accuracy, sensitivity and specificity. Table 1 shows the results in term of accuracy, sensitivity and specificity on the CNN models for "PSB 2015 crowdsourced nuclei" dataset.

Table 1. Result for PSB 2015 crowdsourced nuclei dataset

\begin{tabular}{|c|c|c|c|}
\hline $\begin{array}{c}\text { Image } \\
\text { number }\end{array}$ & $\begin{array}{c}\text { Sensitivity } \\
(\boldsymbol{\%})\end{array}$ & $\begin{array}{c}\text { Specificity } \\
(\boldsymbol{\%})\end{array}$ & $\begin{array}{c}\text { Accuracy } \\
(\boldsymbol{\%})\end{array}$ \\
\hline 1 & 87.58687 & 93.68845 & 92.31737 \\
\hline 2 & 98.61384 & 65.91606 & 72.79952 \\
\hline 3 & 96.79508 & 70.00246 & 77.83639 \\
\hline 4 & 91.73645 & 81.67359 & 83.52104 \\
\hline 5 & 79.54545 & 83.2612 & 82.29236 \\
\hline 6 & 96.16125 & 34.83631 & 58.98791 \\
\hline 7 & 93.27415 & 80.99422 & 84.62668 \\
\hline 8 & 91.1824 & 88.77048 & 89.38857 \\
\hline 9 & 98.01167 & 82.90804 & 85.75924 \\
\hline 10 & 74.17483 & 95.00432 & 89.26411 \\
\hline 11 & 91.61175 & 91.51761 & 91.53721 \\
\hline 12 & 96.57789 & 84.95193 & 86.47826 \\
\hline & & & \\
\hline
\end{tabular}


Table 2. Result for PSB 2015 crowdsourced nuclei dataset

\begin{tabular}{|c|c|c|c|}
\hline $\begin{array}{c}\text { Image } \\
\text { number }\end{array}$ & $\begin{array}{c}\text { Sensitivity } \\
(\%)\end{array}$ & $\begin{array}{c}\text { Specificity } \\
(\%)\end{array}$ & $\begin{array}{c}\text { Accuracy } \\
(\%)\end{array}$ \\
\hline 13 & 98.94195 & 76.51809 & 80.36174 \\
\hline 14 & 98.25697 & 75.66583 & 80.59552 \\
\hline 15 & 95.99198 & 72.59978 & 77.35993 \\
\hline 16 & 98.52066 & 47.47575 & 58.54853 \\
\hline 17 & 99.50254 & 62.37429 & 69.6818 \\
\hline 18 & 98.45196 & 51.96396 & 61.54806 \\
\hline 19 & 97.52604 & 65.30243 & 71.64205 \\
\hline 20 & 85.93293 & 93.1433 & 91.7792 \\
\hline 21 & 90.95585 & 68.27391 & 72.95966 \\
\hline 22 & 79.87251 & 71.18352 & 72.95517 \\
\hline 23 & 87.40971 & 62.23111 & 66.55128 \\
\hline 24 & 66.5609 & 82.35012 & 79.355 \\
\hline 25 & 99.56813 & 50.0477 & 60.36505 \\
\hline 26 & 99.07565 & 52.70402 & 64.58152 \\
\hline 27 & 99.59877 & 62.67468 & 68.31753 \\
\hline 28 & 97.78654 & 57.07224 & 65.34105 \\
\hline 29 & 99.57819 & 26.78715 & 38.09489 \\
\hline 30 & 93.96386 & 75.68104 & 79.43646 \\
\hline 31 & 97.95711 & 61.21888 & 69.29643 \\
\hline 32 & 98.26604 & 62.07793 & 67.70386 \\
\hline 33 & 98.33333 & 63.34946 & 70.90303 \\
\hline 34 & 98.78157 & 31.49814 & 44.2457 \\
\hline 35 & 96.97961 & 55.05537 & 64.88454 \\
\hline 36 & 98.32572 & 69.82065 & 77.04076 \\
\hline 37 & 98.91471 & 55.06906 & 64.13061 \\
\hline 38 & 98.56884 & 55.34972 & 65.79132 \\
\hline 39 & 98.0142 & 49.36744 & 61.17369 \\
\hline 40 & 98.65579 & 66.67912 & 73.57501 \\
\hline 41 & 99.12715 & 67.38293 & 75.76801 \\
\hline 42 & 93.70471 & 82.7632 & 84.31996 \\
\hline 43 & 97.55909 & 64.99771 & 74.21272 \\
\hline 44 & 80.65161 & 75.52523 & 76.45756 \\
\hline 45 & 97.65271 & 65.31935 & 72.254 \\
\hline 46 & 92.36079 & 86.34099 & 87.08305 \\
\hline 47 & 90.95604 & 87.65426 & 88.5743 \\
\hline 48 & 98.45531 & 70.55847 & 78.51481 \\
\hline 49 & 97.66016 & 79.31877 & 83.33451 \\
\hline 50 & 76.86213 & 89.20831 & 85.79382 \\
\hline
\end{tabular}

The accuracy, sensitivity and specificity, on test images are represented graphically in Figure 8.

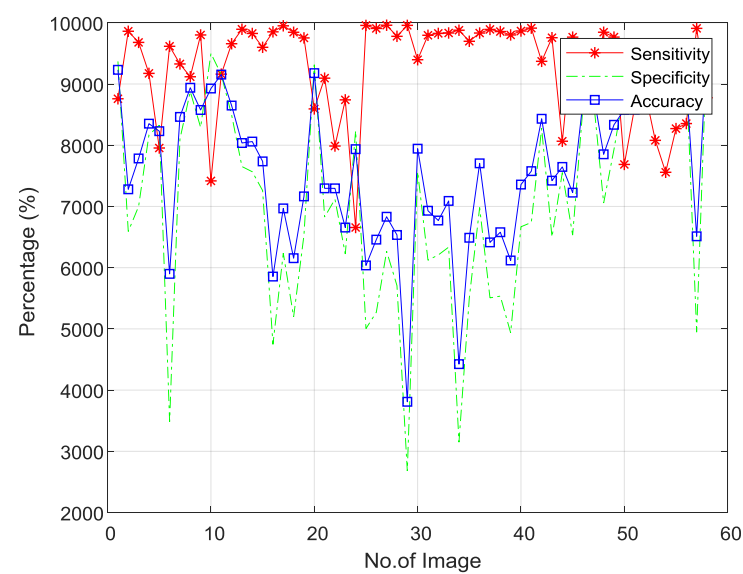

Fig 8: Sensitivity, specificity and accuracy of our model

The results, original image, ground truth image and result of the semantic segmentation are shown in Figure 9.

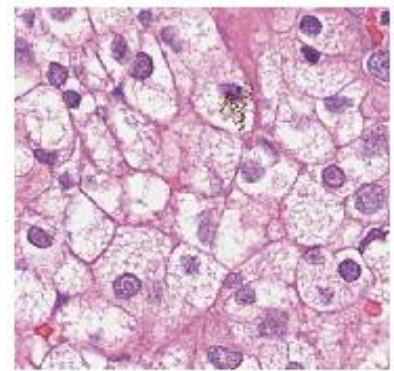

(a)

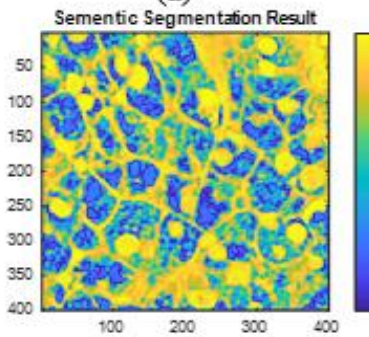

(c)

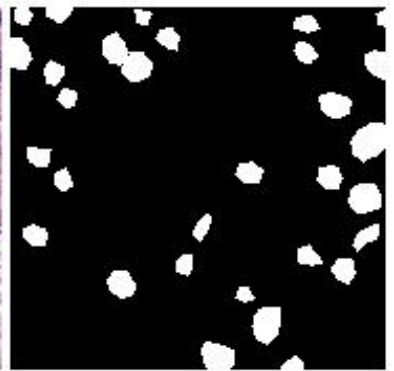

(b)

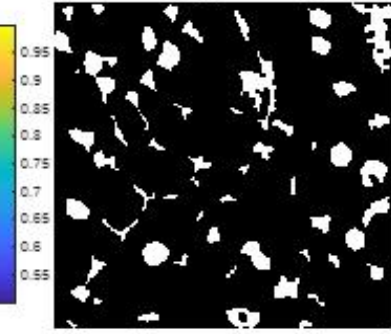

(d)
Fig 9: Results, a) Original image, b) Ground truth image, c) result of the semantic segmentation, d) After binarization

The segmentation process can be formulated as an optimization problem. The designed problem can be considered as determining the optimal values of the algorithm parameters for a successful segmentation result or finding the optimal segmentation between possible segmentations. Pixels were separated from each other by modeling herd behaviors using optimization algorithms based on herd intelligence and the desired regions were revealed.

Table 2 shows the resulted image at the end of each step of applying Artificial Bee Colony (ABC) for cancer nuclei detection. $\mathrm{ABC}$ is an optimization technique based on herd intelligence [29]. Thus, shape and textural features are removed to provide better mitotic cell detection capability. Four histological images were stochastic selected and the detection process was done in conjunction with the proposed method. The results showed that the presented method had superior sensitivity than any other method in the literature. 
Table 2. Four images showing based cancer nuclei detection using artificial bee colony algorithm

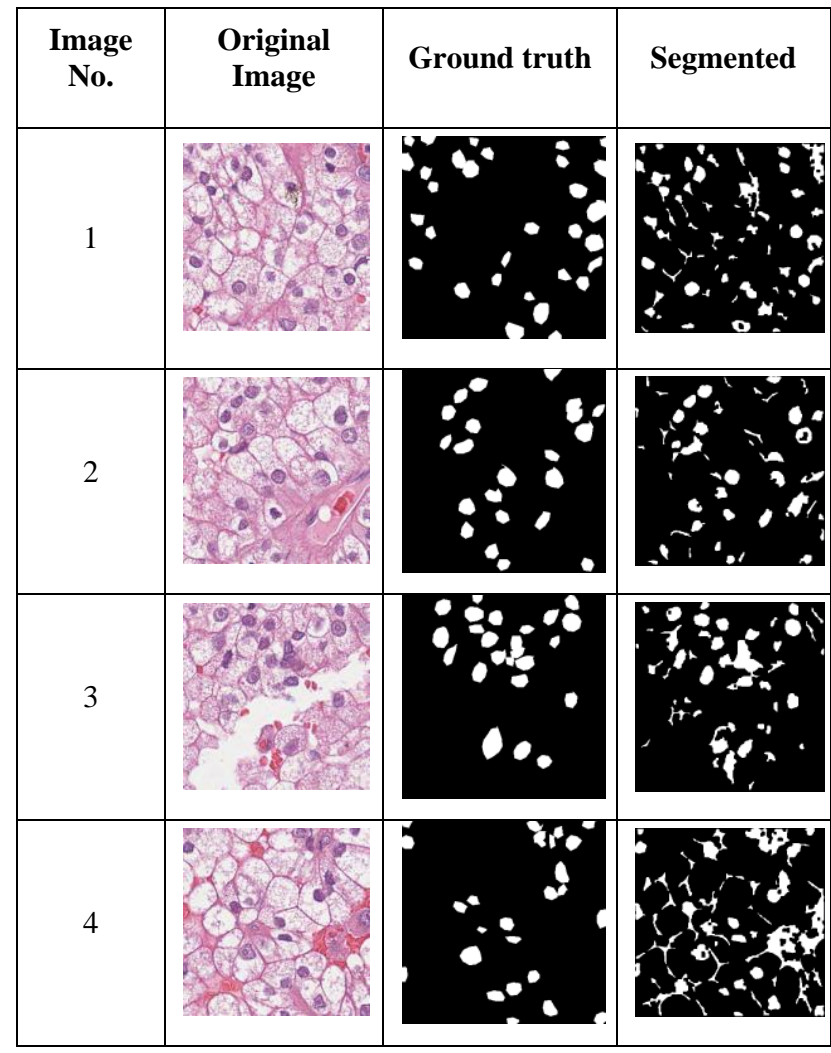

The comparison between proposed method and other method is shown in Table 3 .

Table 3. Performance parameter results of the 810 histological images from PSB 2015 crowdsourced nuclei dataset

\begin{tabular}{|c|c|c|c|}
\hline Method & Precision & Recall & Accuracy \\
\hline SC-CNN [8] & 0.758 & 0.827 & 0.791 \\
\hline SSAE [30] & 0.617 & 0.644 & 0.630 \\
\hline CRImage [31] & 0.657 & 0.461 & 0.542 \\
\hline LIPSyM [24] & 0.725 & 0.517 & 0.604 \\
\hline SR-CNN [32] & 0.783 & 0.804 & 0.793 \\
\hline Proposed Method & $\mathbf{0 . 8 4 4}$ & $\mathbf{0 . 8 3 2}$ & $\mathbf{0 . 8 5 1}$ \\
\hline
\end{tabular}

The results were compared with the segmentation results obtained from the CP-CNN, SSAE, CRImage, LPSyM, SR$\mathrm{CNN}$ algorithms under the same conditions. The successful results display that the proposed method has a consistent accuracy for semantic image segmentation. As a result, the CNN model gave the highest performance with precision (0.844), recall (0.832) and accuracy $(0.851)$. As a result of the experimental study, precision $(0.844)$, recall $(0.832)$ and accuracy $(0.851)$ parameters were obtained.

\section{CONCLUSION}

In this study, a deep learning approach is proposed for the semantic segmentation study of breast cancer images. The development process was carried out in five basic steps: Initialization, implementation of $\mathrm{CNN}$, morphological operations, binarization and segmentation and accuracy of nucleus segmentation. Convolutional Neural Network based on detection and semantic segmentation of cell nuclei for breast cancer was performed on the "PSB 2015 crowdsourced nuclei" data set. As a result, the CNN model gave the highest performance with precision (0.844), recall (0.832) and accuracy (0.851) compared to other classifiers in the literature and the most advanced methods. The precision, recall and accuracy rates obtained as a result of this study have been observed to be successful enough.

\section{REFERENCES}

[1] Gao, X., Li, W., Loomes, M., and Wang, L., 2017. A fused deep learning architecture for viewpoint classification of echocardiography, Information Fusion, vol. 36, 103-113.

[2] Shwendicke, F., Golla, T., Dreher, M., and Krois, J., 2019. Convolutional neural networks for dental image diagnostic: A scoping review, Journal of Dentistry, vol. 91 .

[3] Voulodimos, A., Doulamis, N., Doulamis, A., and Protopapadakis, E., 2018. Deep learning for computer vision: A brief review. Computational Intelligence and Neuroscience, vol. 2018

[4] Zhao, R., Yan, R., Chen, Z., Mao, K., Wang, P., and Gao, R. X., 2019. Mechanical systems and signal processing. Mechanical systems and signal processing, vol. 115, 213-237.

[5] Zeebaree, D. Q., Haron, H., Abdulazeez, A. M., and Zebari, D., A., 2019. Machine learning and region growing for breast cancer segmentation. International Conference on Advanced Science and Engineering.

[6] Rakhlin, A., Shvets, A., Iglovikov, V., and Kalinin, A. A. 2018. Deep convolutional neural networks for breast cancer histology image analysis. International Conference Image Analysis and Recognition, 737-744.

[7] Lévy, D., and Jain, A. 2016. Breast mass classification from mammograms using deep convolutional neural networks. arXiv preprint arXiv:1612.00542.

[8] Cruz-Roa, A. et al., 2014. Automatic detection of invasive ductal carcinoma in whole slide images with convolutional neural networks. Medical Imaging 2014, vol. 9041

[9] Sirinukunwattana, K., et al., 2016. Locality sensitive deep learning for detection and classification of nuclei in routine colon cancer histology images. IEEE Trans. Med. Imaging, vol. 35, no. 5, 1196-1206.

[10] Wang, H., et al., 2014. Cascaded ensemble of convolutional neural networks and handcrafted features for mitosis detection. Medical Imaging 2014, vol. 9041.

[11] Cruz-Roa, A. A., Ovalle, J. E. A., Madabhushi, A., and Osorio, F. A. G., 2013. A deep learning architecture for image representation, visual interpretability and automated basal-cell carcinoma cancer detection. International Conference on Medical Image Computing and Computer-Assisted Intervention, 403-410. 
[12] Wan, T., Cao, J., Chen, J., and Qin, Z., 2017. Automated grading of breast cancer histopathology using cascaded ensemble with combination of multi-level image features. Neurocomputing, vol. 229, 34-44.

[13] Cireşan, D. C., Giusti, A., Gambardella, L. M., and Schmidhuber, J., 2016. Mitosis detection in breast cancer histology images with deep neural networks. International Conference on Medical Image Computing and Computer-assisted Intervention, 411-418.

[14] Irshad, H., et al., 2014. Crowdsourcing image annotation for nucleus detection and segmentation in computational pathology: evaluating experts, automated methods, and the crowd. Pacific symposium on biocomputing Cochairs, World Scientific, 294-305.

[15] Beck Lab., 2020. PSB Crowdsourced Nuclei Annotation, https://becklab.hms.harvard.edu/software/psbcrowdsourced-nuclei-annotation-data-1.

[16] Ghosh, S., Das, N., Das I., and Maulik, U., 2019. Understanding deep learning techniques for image segmentation. ACM Computing Surveys, vol. 52, no. 4.

[17] Fawcett, T., 2006. An introduction to ROC analysis. Pattern recognition letters, vol. 27, no. 8, 861-874.

[18] Story, M., and Congalton, R. G., 1986. Accuracy assessment: A user's perspective. Photogramm Eng. Remote Sensing, vol. 52, no. 3, 397-399.

[19] Rahebi, J., and Hardalaç, F., 2014. Retinal blood vessel segmentation with neural network by using gray-level co-occurrence matrix-based features. Journal of medical systems, vol. 38 , no. 8 .

[20] Fraz, M. M., et al., 2012. Blood vessel segmentation methodologies in retinal images-a survey. Computer methods and programs in biomedicine, vol. 108, no. 1, 407-433.

[21] Diakogiannis, F., Waldner, F., Caccetta, P., and Wu, C., 2020. ResUNet-a: A deep learning framework for semantic segmentation of remotely sensed data. ISPRS Journal of Photogrammetry and Remote Sensing, vol. $162,94-114$

[22] WR. Crum, Camara, O., and Hill, DL, 2006. Generalized overlap measures for evaluation and validation in medical image analysis. IEEE transactions on medical imaging, 1451-1461.

[23] Sudre, C. H., Wenqi, L., Vercauteren, T., Ourselin, S., and Cardoso, M. J., 2017. Generalised Dice overlap as a deep learning loss function for highly unbalanced segmentations. Deep Learning in Medical Image Analysis and Multimodal Learning for Clinical Decision Support, 240-248.

[24] Kuse, M. Wang, Y.-F., Kalasannavar, V., Khan, M., and Rajpoot, N., 2011. Local isotropic phase symmetry measure for detection of beta cells and lymphocytes. Journal of pathology informatics, vol. 2.

[25] Ding, S., Li, H., Su, C., and Yu, J., 2013. Evolutionary artificial neural networks: A review. Artificial Intelligence Review, vol. 39, no. 3.

[26] Bengio, Y., Goodfellow, I., and Courville, A., 2016. Convolutional Networks. An MIT Prees Book, Chapter 9.

[27] Serra, J., 1986. Introduction to Mathematical Morphology. Computer Vision, Graphics and Image Processing, 62-66.

[28] Niea, F., Zhangb, P., Lia, J., and Din, D., 2017. A novel generalized entropy and its application in image thesholding. Signal Processing, 23-34.

[29] Karaboga, D., 2005. An idea based on honey bee swarm for numerical optimization, Technical Report TR06, Erciyes University, Engineering Faculty Computer Engineering Department.

[30] Xu, J., et al., 2015. Stacked sparse autoencoder (SSAE) for nuclei detection of breast cancer histopathology images, IEEE transactions on medical imaging, vol. 35, no. $1,119-130$

[31] Yuan, Y., et al., 2012. Quantitative image analysis of cellular heterogeneity in breast tumors complements genomic profiling. Science translational medicine, vol. 4 , no. 157.

[32] Xie, Y., Xing, F., Kong, X., Su, H., and Yang, L., 2015. Beyond classification: structured regression for robust cell detection using convolutional neural network. International Conference on Medical Image Computing and Computer-Assisted Intervention, 358-365. 